Supporting Information for:

\title{
Functional wood-foam composites for controlled uptake and release
}

Tamara L. Church, ${ }^{a}$ Konstantin Kriechbaum, ${ }^{a}$ S. Noushin Emami, ${ }^{b, c}$ Raimondas Mozūraitis, ${ }^{d}$ and Lennart Bergström ${ }^{a, *}$

${ }^{a}$ Department of Materials and Environmental Chemistry, Stockholm University, Stockholm, 10691 Sweden

${ }^{b}$ Department of Molecular Biosciences, Wenner-Gren Institute, Stockholm University, Stockholm, Sweden

${ }^{c}$ Natural Resources Institute, FES, University of Greenwich, London, UK

${ }^{d}$ Department of Zoology, Stockholm University, Stockholm, SE-10691, Sweden

* Corresponding author: lennart.bergstrom@mmk.su.se

Number of pages: 6

Number of figures: 9

Number of tables: 0 
Figure S1. Impact of tannic acid on foam volume

Figure S2. Fluorescence microscopy images of $\mathbf{C N C}_{\mathrm{NB}}-\mathbf{M C}_{\mathrm{DTAF}}-\mathrm{TA} / \boldsymbol{P}$. abies $\quad$ S3

Figure S3. (CNC-MC-TA) aq foam after rotor-stator mixing $\quad$ S3

Figure S4. SEM images of a vacuum-infiltrated wood-foam composite, CNC-MC-TA/P. abies $_{\text {vacuum }} \quad$ S4

Figure S5. Wide-angle X-ray diffraction patterns of P. abies and CNC-MC-TA/P. abies 20k-5 $\quad$ S4

Figure S6. $\mathrm{N}_{2}$ sorption isotherms of CNC-MC-TA/P. abies $20 \mathrm{k}-30$ and TCNF-B-SEP/P. abies $20 \mathrm{k}-30 \quad$ S4

Figure S7. $\mathrm{CO}_{2}$ uptake by $\mathbf{C N C}-\mathbf{M C}-\mathbf{T A}-\mathbf{N H}_{2}$ foam $\quad$ S5

$\begin{array}{ll}\text { Figure S8. Examples of images used to calculate filled/empty tracheids } & \text { S5 }\end{array}$

Calculation of shear rates in the rotor-stator mixer (including Figure S9) $\quad$ S6

(a)

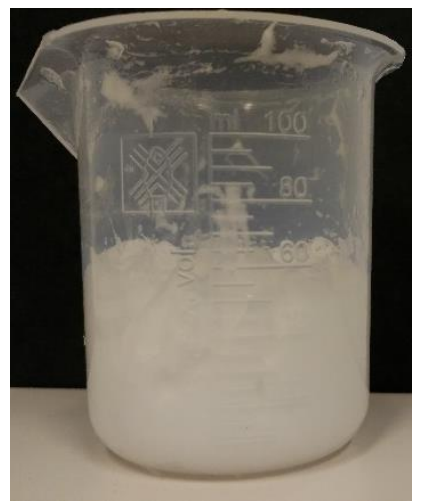

(b)

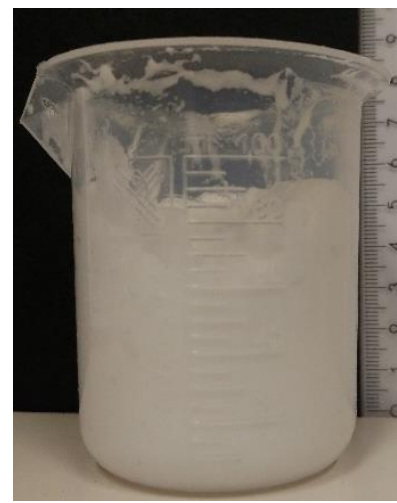

Figure S1. Photographs showing foam volume during the synthesis of (CNC-MC-TA) aq. (a) Aqueous suspension of cellulose nanocrystals and methylcellulose after high-shear foaming with a rotor-stator mixer for $3 \mathrm{~min}$ at $10000 \mathrm{rpm}$. (b) The same foam after a solution of tannic acid was added dropwise while foaming with a high-shear rotor-stator mixer at $10000 \mathrm{rpm}$. 
(a) CNC-MC-TA/P. abies $10 \mathrm{k}-5$

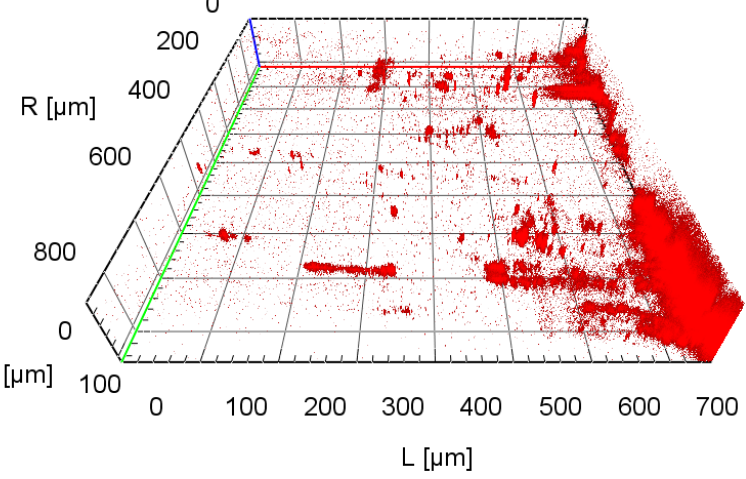

(b) CNC-MC-TA/P. abies $10 \mathrm{k}-10$

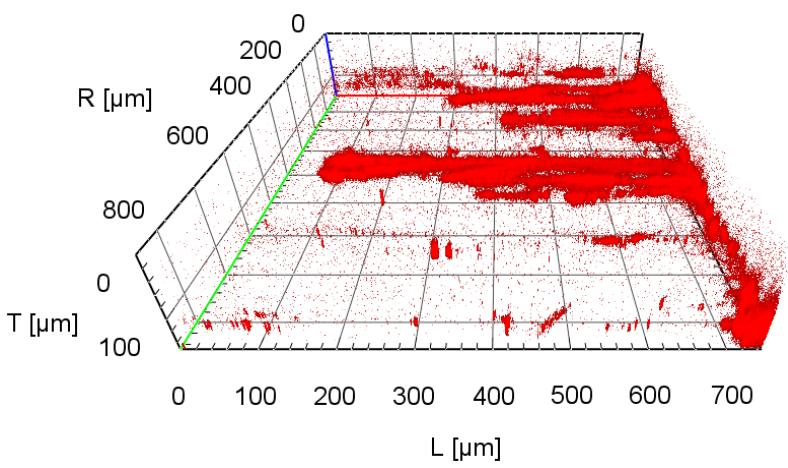

(c) CNC-MC-TA/P. Abies2ok-5

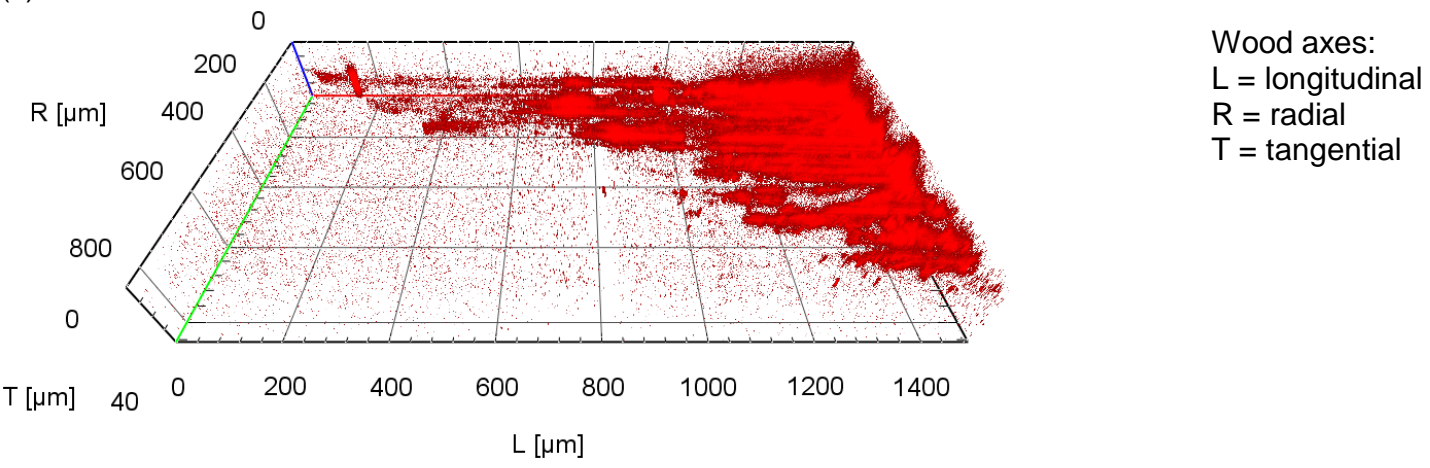

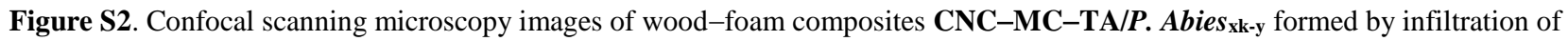
fluorescently labelled $\left(\mathbf{C N C}_{\mathbf{N B}}-\mathbf{M C}_{\mathbf{D T A F}}-\mathbf{T A}\right)_{\text {aq }}$ into $P$. abies using a the shear forces generated by a rotor-stator mixer operated $10^{3} \mathbf{x}$ rpm for $\mathbf{y}$ min. Freeze-dried samples were sliced open longitudinally and imaged using $\lambda_{\text {excitation }}=633 \mathrm{~nm}$ and $\lambda_{\text {detection }}=$ 638-759 nm. Foam ingress into the wood is at right in each image. (a) CNC-MC-TA/P. Abies 10k-5. (b) CNC-MC-TA/P. Abies 10k-10. (c) CNC-MC-TA/P. Abies 20k-5.

(a)
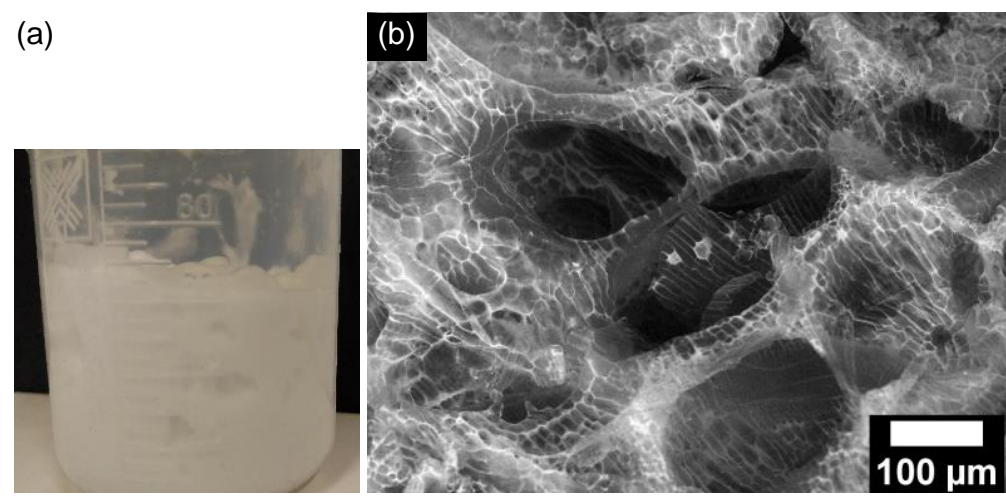

(d)

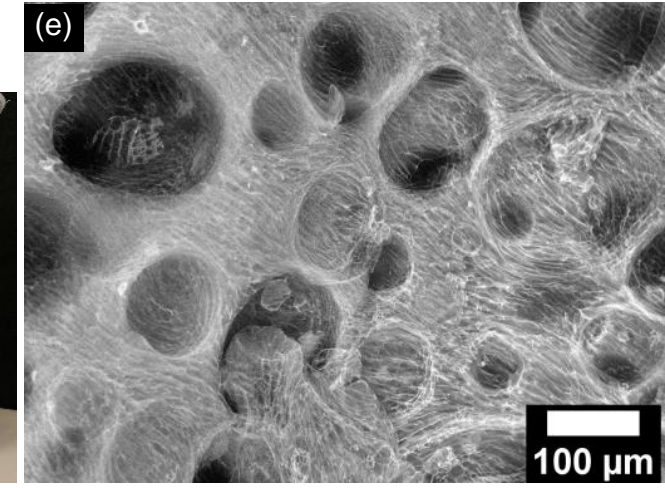

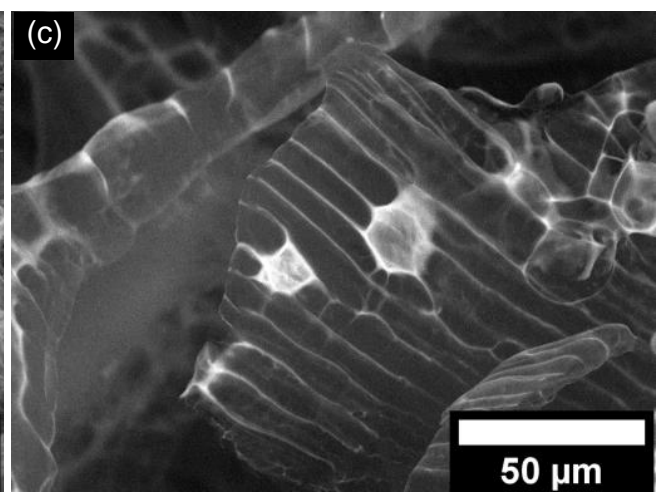

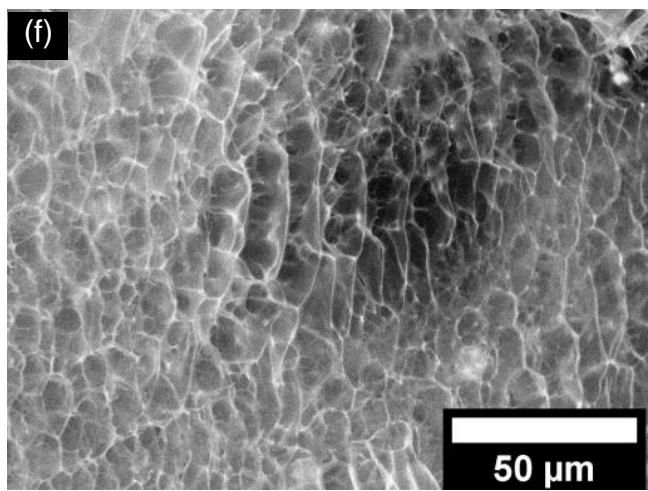

Figure S3. Images of foams composed of cellulose nanocrystals, methylcellulose, and tannic acid after rotor-stator mixing. (a) Digital photograph of wet (CNC-MC-TA)aq foam after high-shear mixing for $1 \mathrm{~min}$ at $10000 \mathrm{rpm}$, and $(\mathrm{b}, \mathrm{c})$ scanning electron microscope images of the foam after freezing in $\mathrm{N}_{2}(\mathrm{l})$ and freeze-drying. (d) Digital photograph of wet (CNC-MC-TA) aq foam after high-shear mixing for $1 \mathrm{~min}$ at $20000 \mathrm{rpm}$, and (e,f) scanning electron microscope images of the foam after freezing in $\mathrm{N}_{2}(\mathrm{l})$ and freeze-drying. 

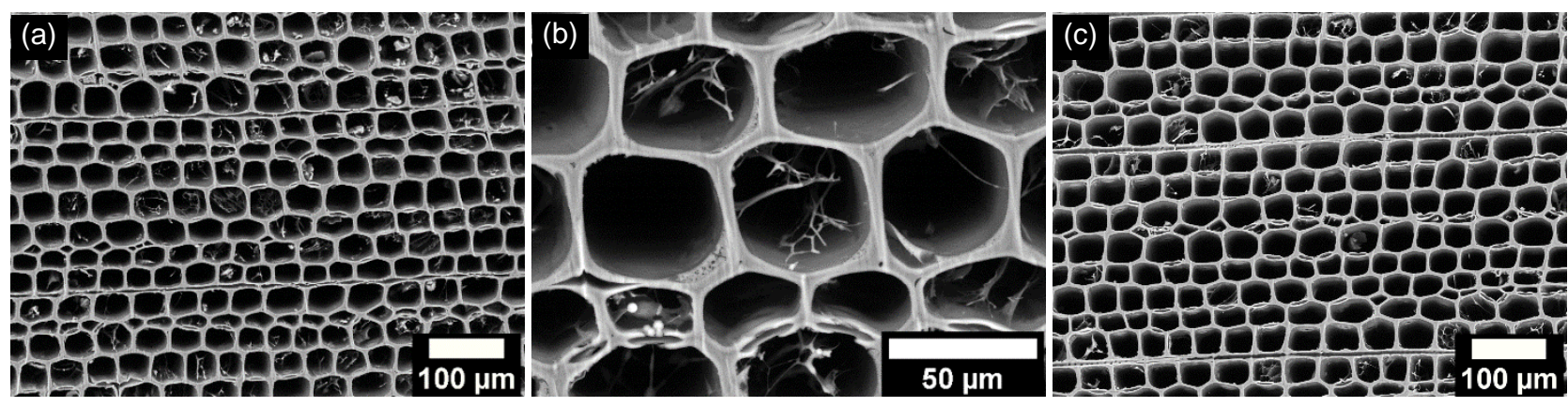

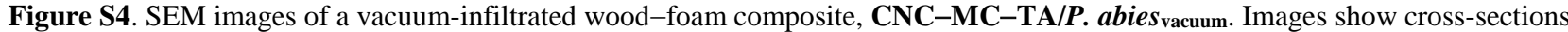
$(a, b) 100$ and (c) $200 \mu \mathrm{m}$ deep in the composite.
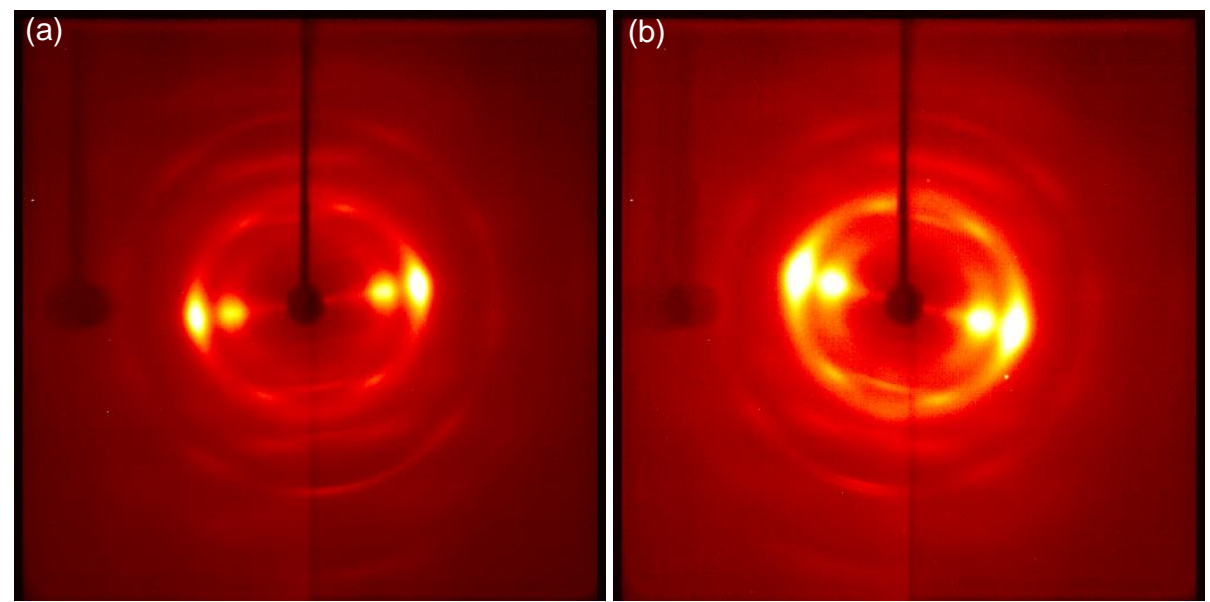

Figure S5. Wide-angle X-ray diffraction patterns of (a) P. abies and (b) CNC-MC-TA/P. abies20k-5. Samples were mounted at a $10-15^{\circ}$ angle to the goniometer head, and irradiated with $\mathrm{Cu} \mathrm{K \alpha}$ radiation $(\lambda=0.154 \mathrm{~nm})$. Data was collected on a CCD camera positioned $45 \mathrm{~mm}$ from the goniometer.

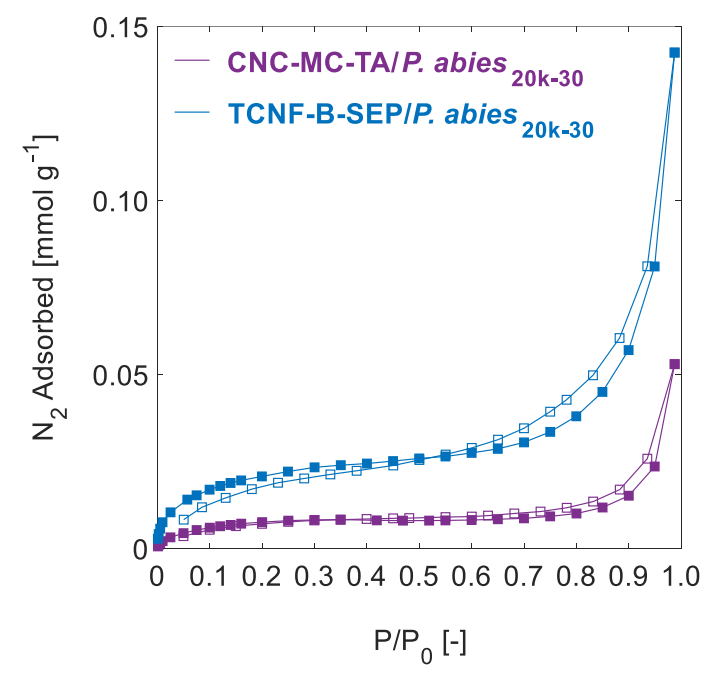

Figure S6. $\mathrm{N}_{2}$ adsorption and desorption isotherms for CNC-MC-TA/P. abies $20 \mathrm{k}-30$ and TCNF-B-SEP/P. abies 20k-30, measured at $-196{ }^{\circ} \mathrm{C}$. Samples were degassed under vacuum at $90{ }^{\circ} \mathrm{C}$ for $40 \mathrm{~h}$ prior to measurement. 


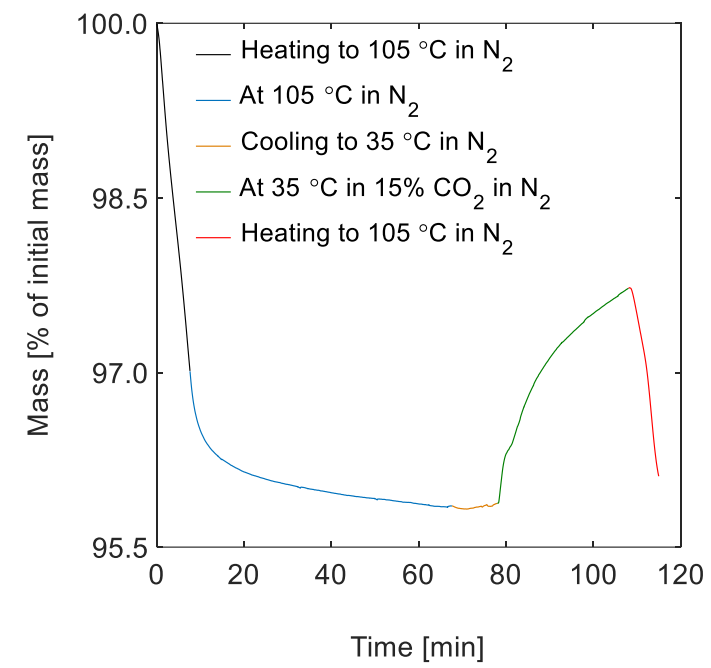

Figure S7. $\mathrm{CO}_{2}$ uptake by unconfined freeze-dried $\mathbf{C N C}-\mathbf{M C}-\mathbf{T A}-\mathbf{N H}_{2}$ foam.
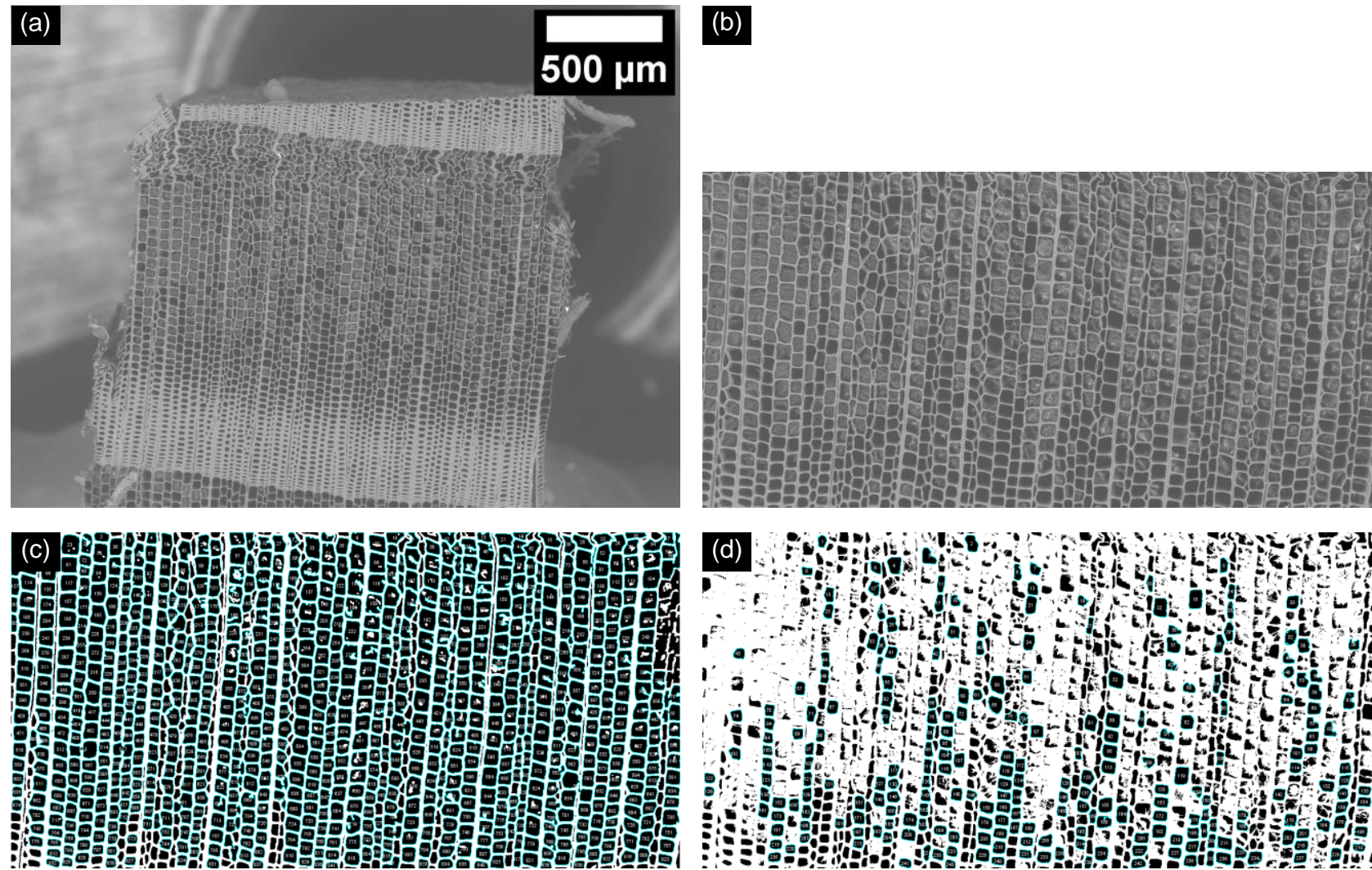

Figure S8. Example of images created in the Fiji implementation of Image ${ }^{54,55}$ to calculate the fraction of early wood tracheids that are filled with foam. (a) SEM image of CNC-MC-TA/P. abies 20k-5, including scale bar; (b) the same image, rotated and cropped to show only early wood. (c) The cropped image, after converting to binary with $23 \%$ of pixels being over the threshold, then performing the 'Open' operation twice and 'Erode' once, and finally counting the dark regions having size $\geq 500 \mu \mathrm{m}^{2}$ and circularity $\geq 0.1$. The overlay mask (light blue) shows the cells counted by the procedure. (d) The cropped image, after converting to binary with $70 \%$ of pixels above the threshold, then counting the dark regions having size $\geq 500 \mu \mathrm{m}^{2}$ and circularity $\geq 0.5$. The overlay mask (light blue) shows the empty cells counted by the procedure. The total number of empty cells was manually adjusted to include the empty cells in the bottom left corner that were not counted by ImageJ. The filled cells are given by the difference between the counts in (c) and (d). 


\section{Calculation of Shear Rate Between the Rotor and Stator}

The shear rate between the rotor and the stator can be calculated for a point between the rotor and a tine of the stator (Figure S8). The shear rate $\gamma$ is related to the velocity at the rotor, $v$, and the separation $h$ between the rotor and stator, by

$\gamma=\frac{v}{h}$

The flow velocity tangential to the rotation of the rotor can be calculated from the outer diameter of the rotor, $d_{\mathrm{R}, \text { outer }}$, and the rotation rate $\omega$ of the rotor (in rotations per minute). The separation between the rotor and stator is the absolute difference between the outer diameter of the rotor and the inner diameter of the stator $d_{\text {s,inner, so }}$

$\gamma=\frac{\pi \omega d_{R, \text { outer }}}{60\left(d_{S, \text { inner }}-d_{R, \text { outer }}\right)}$

Six measurements (three each on two different rotors) gave $d_{\mathrm{R} \text {,outer }}=12.64 \pm 0.01$, and six measurements (three each on two different stators) gave $d_{\mathrm{s} \text {,inner }}=16.0 \pm 0.2$. The rotation rate could vary from the set point by up to $400 \mathrm{rpm}$, so $\omega=10000 \pm 400$ or $20000 \pm 400$ rpm, which gives $\gamma=(3900 \pm 200)$ or $(7900 \pm 300) \mathrm{s}^{-1}$.

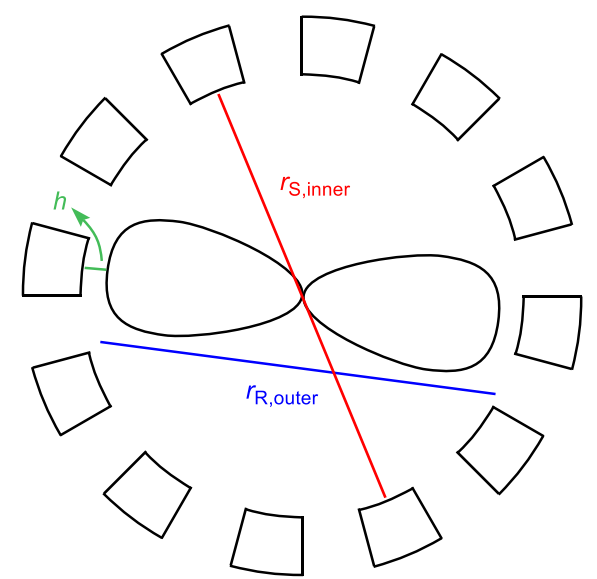

Figure S9. Diagram of the rotor-stator, seen from below, indicating the measurements used to calculate the shear rate between the rotor and stator. Not to scale. 